\title{
On the thermodynamics of listric faults
}

\author{
Klaus Regenauer-Lieb ${ }^{1,2}$, Bruce Hobbs ${ }^{2}$, and Alison Ord ${ }^{2}$ \\ ${ }^{1}$ Johannes Gutenberg-Universität Mainz, Geophysics and Geodynamics, 55099 Mainz, Germany \\ ${ }^{2}$ CSIRO Exploration and Mining Perth (ARRC), PO Box 1130, Bentley WA 6102, Australia
}

(Received June 15, 2004; Revised November 21, 2004; Accepted December 4, 2004)

\begin{abstract}
We investigate a novel fully coupled thermal-mechanical numerical model of the crust in order to trace the physics of interaction of its brittle and ductile layers. In a unified approach these layers develop in a natural transition as a function of the state variables pressure, deviatoric stress, temperature and strain-rate. We find that the main storage of elastic energy lies in the domain where brittle and ductile strain-rates overlap so that shear zones are attracted to this zone of maximum energy dissipation. This dissipation appears as a local heat source (shear heating). The brittle-ductile transition zone evolves through extreme weakening by thermo-mechanical feedback. The physics of the weakening process relies on repeated breaching of a critical energy flux threshold for feedback within this sub-horizontal brittle-ductile flow layer, thus developing unstable slipping events at postand pre-seismic strain-rates. The width- and the temperature domain of the feedback layer is controlled by the activation enthalpy $Q$ of the material. For olivine rheology $(Q \sim 500 \mathrm{~kJ} / \mathrm{mol})$ the layer can be extremely thin $<500 \mathrm{~m}$ and adheres to the $875 \mathrm{~K}$ isotherm. For quartz $(Q \sim 135 \mathrm{~kJ} / \mathrm{mol})$ the width of the feedback layer fans out into multiple interacting ductile faults covering a temperature domain of 450-600 K. The weakening by thermalmechanical feedback entirely controls the location and rejuvenation of upper crustal shear zones propagating from the detachment upwards in the form of listric faults. Within the detachment shear layer we identify an astonishing rich dynamics featuring distinct individual creep bursts. We argue that the rich ductile dynamics holds the key to earthquakes in the brittle field.
\end{abstract}

Key words: Quartz rheology, geodynamic modelling, brittle-ductile transition, crustal extension.

\section{Introduction}

Slip and flow processes below the seismogenic region have traditionally been interpreted in terms of a smooth viscous flow potential (Lyakhovsky et al., 2001) which does not feature flow localization phenomena. In these models the viscous substratum below the seismogenic region acts like a slave to the brittle processes with an effective damping effect on the vigour of seismic instabilities. Such an approach allows insights into the elasto-dynamics of the brittle crust at and around the point of the occurrence of an earthquake, but its use for time scales longer than the earthquake is limited. At long geological time scale on the other hand it has been a common practice to neglect the brittle layer and deal with the constraints from viscous flow laws and their potential for flow localization (Regenauer-Lieb and Yuen, 2003). In these models the brittle crust is viewed as insignificant to control slip in the ductile part of the lithosphere. Both contradictory approaches are justified at their particular pre-set time scale but we are still lacking an understanding of what happens in between. Prior and after the earthquakes we also expect some dynamics owing to the feedback between brittle and ductile regimes. We are presenting here a first attempt of looking at combined ductile and brittle dynamics, focussing on the effect of thermal-mechanical instabilities. We investigate the "bottom up" dynamics of

Copy right(c) The Society of Geomagnetism and Earth, Planetary and Space Sciences (SGEPSS); The Seismological Society of Japan; The Volcanological Society of Japan; The Geodetic Society of Japan; The Japanese Society for Planetary Sciences; TERRAPUB. the fully coupled intrinsic brittle-ductile feedback loop. The possibility of altering the steady flow cycle between seismic events and thermal-mechanical instabilities leads to the events. In doing so we derive a simple solution to the emergence of listric faults out of self-organization of ductile instabilities below the seismogenic zone. We present the first numerical results illustrating the early postulates for shear zone development and detachment faulting (Hobbs et al., 1986; Ogawa, 1987; Ord and Hobbs, 1989; Yuen et al., 1978) and discuss earthquake genesis coupled to thermalmechanical instabilities.

After giving a brief description of the numerical approach we will, in the sections to come, first discuss: a) the classical linear stability analyses and the critical temperature for instability as well as its implication for detachment shear zone and weakening on shear zones. We conclude this section in pointing out the loose ends of linear stability analyses; b) We briefly give an introduction to a thermodynamic theory introducing the concept of critical dissipation; c) We go on and apply the theory to discuss the development of listric faults in the continental crust and; d) We conclude with a brief discussion of implications for the genesis of earthquakes and point out the necessity for future investigations.

\section{Methodology}

We use a commercial Finite Element Code (ABAQUS) which is particularly suited to solve strongly non-linear problems such as fracture mechanics. Hence, the code pro- 
vides a good basis to solve the difficult problem of nonlinear interactions. Our method to extend the capabilities to geological problems relies on an implementation described elsewhere (Regenauer-Lieb and Yuen, 2004). We give a brief outline of the method.

The method relies on solving the rheologymomentum-energy feedbacks in a fully coupled thermal-mechanical framework. We use an adaptive implicit time stepping scheme designed for accurately tracking the energy fluxes during and after material instabilities. This scheme consists of a simultaneous solution of the energy and momentum equations. The underlying thermodynamic approach for mechanical systems out of equilibrium is probed in the evolution of the internal minus the dissipated energies (Helmholtz free energy) by dynamically adapting time steps.

\section{Linear Stability Analyses and Thermal- Mechanical Instabilities}

Two physical processes are essential for thermalmechanical instabilities, one process is the energy stored during deformation and the other the energy dissipated. The rock matrix may store energy as it strains in microstructural defects such as brought about by dislocations rearrangements, micro-cracks, change in grain size or other microstructural processes. The rock matrix thus experiences a so-called strain hardening, mathematically expressed as the partial derivative of the stress over the strain. The second process is the dissipative work, which appears as heat (with an efficiency factor $\chi$ of less than but close to unity) in turn weakening the rheology. Quasi-static equilibrium in the momentum equation can be achieved if the strain hardening is larger than the thermal softening. On the other hand the same linear stability analysis predicts a finite amplitude instability if strain hardening is less than the thermal weakening of the rock matrix due to dissipation. Thermal weakening of the rock matrix increases with decreasing temperature (see Eq. (2)) thus the criterion boils back to a critical value at or below a critical temperature $T_{c}$

$$
\frac{\partial \sigma}{\partial \varepsilon}<\frac{Q}{n R T_{c}^{2}} \frac{\chi \sigma^{2}}{\rho c_{p}}
$$

where $Q$ is the activation enthalpy, $n$ is the power law exponent, $R$ is the universal gas constant, $\rho$ is the density and $c_{p}$ is the specific heat (Hobbs and Ord, 1988; Hobbs et al., 1986). This is the essential criterion for thermalmechanical instability in quasi-adiabatic deformation for elasto-visco-plastic materials. It has been formulated for mechanical stability/instability of any point in space. In 2 or 3 dimensions it is concomitant with the appearance of a shear zone out of homogeneous flow.

For deformation in the fully ductile regime (e.g. inside the convecting mantle) the efficiency of shear heating is frequently set to $\chi=1$ and deformation is modelled by viscous behaviour. Ogawa (Ogawa, 1987) considered viscoelasticity and derived the same criterion (his equation 30) for the nucleation of a shear zone. In a one-dimensional form the stiffness of reduction of the growing shear zone is also influenced by the width of the shear zone. The stiffness reduction due to thermal-mechanical feedback is obtained by dividing the term on the right side of the inequality in Eq. (1) by the width of the shear zone. Wide shear zones obviously have a stronger strength reduction than narrow shear zones, i.e. they require a smaller amount of shear heating to become unstable. We will come back to this point later in the discussion. Ogawa and Hobbs et al. point out that their linear stability analysis only gives a criterion for instability but says nothing about the evolution of the shear zone after instability.

However, by resorting to a simple viscous approximation a very similar relation can be formalized for the weakening on the shear zone caused by thermal mechanical feedback. Assuming $\chi=1$ the reduction in viscosity is found to be approximately described by

$$
\eta \approx 8 \kappa \rho c_{p} \frac{R T_{c}^{2}}{Q v^{2}}
$$

$\eta$ is the viscosity on the shear zone, $\kappa$ is the thermal diffusivity $v$ is the velocity of instability (Yuen et al., 1978). The equation is exact for Newtonian viscous flow $(n=$ 1) and a good approximation for power law viscous flow (Fleitout and Froidevaux, 1980). Note the symmetry between thermal-mechanical weakening and viscosity reduction in Eq. (1) and (2). Replacing the (state) variable stress by the (state) variable velocity equation 2 comes out of 1 in a reciprocal form if multiplied by a constant times the diffusivity. A higher activation energy hence produces a more vigorous instability in terms of stress derivatives and also reduces the viscosity on the shear zone more significantly. Similarly if the energy in the system is high enough for thermal-mechanical instabilities to occur at lower temperatures they will be generally stronger in terms of weakening and stress release.

In geology the stress-strain rate level that can be reached through thermal-mechanical feedback is kinematically limited in the far field by the plate velocities in turn stabilized by viscous dissipation in lithosphere-mantle coupling through convection. Another stress limiter is given by potential brittle failure providing a limiting cap to thermalmechanical instabilities at higher stress level. The strong dependence of thermal-mechanical feedback on the activation enthalpy $Q$ expressed in Eq. (1) and (2) implies material characteristic critical isotherms. When applying a constant far field velocity to a vertically stratified plate with rocks of different $Q$ the condition for thermal-mechanical instabilities will therefore create several weak subhorizontal detachments for horizontal isotherms at several different critical temperatures being material characteristics of the particular layer. For velocities that are too low these detachments may not develop because they are below the critical shear heating level defined in Eq. (1). We suggest that this is a basic mechanism underlying the formation of listric faults and it has indeed been suggested that such instabilities create distinct weak detachments at different critical isotherms for e.g. quartz, feldspar and olivine rheology (Ord and Hobbs, 1989).

While the analytical approaches based on linear stability analyses give insight into the potential physics operating in geological shear zones there are also a couple of loose ends that cannot be covered without testing the hypothesis in a 
numerical model. As already pointed out the approach cannot describe processes that happen during or subsequent to localization. As such, the questions whether the instability can go seismic and how high the temperature rises or if it only occurs in a mild form, does it cause sufficient weakening to cause subhorizontal detachments, remained entirely open issues. Note also, that such full dynamic mapping in hypothetical 1-D and 2-D numerical experiments remained elusive for a long time until computationally demanding extremely high temperature, temporal and spatial resolution were achieved (Cherukuri and Shawki, 1995a; Cherukuri and Shawki, 1995b; Kameyama et al., 1999; Kameyama et al., 1997; Ogawa, 1987; Regenauer-Lieb and Yuen, 1998). Perhaps the most profound criticism to the linear stability analyses is that it is a local criterion for a single hypothetical fault and not a global criterion for multiple interacting faults. This does not change the basic conclusions but calls for a full thermodynamic framework.

\section{Thermodynamic Framework}

The analysis can be cast into a thermodynamic framework when stability/instability is understood in terms of a balance of energy fluxes for an elastic reference volume and not just in terms of an equilibrium of forces. Another addition is that the energy framework gives an accurate description of what happens after instability, because the weakening is controlled by the energy state within the shear zone and its exchange with the surroundings. We are presenting here a much abridged and simplified version of the full energy approach to shear zones (Regenauer-Lieb and Yuen, 2003), outlining only its key elements. The second law of thermodynamics implies that for the deformation of a specific reference volume the dissipation must be positive

$$
D_{i} \equiv \sigma_{i j} \dot{\varepsilon}_{i j}-\rho \frac{\partial \psi}{\partial q_{\alpha}} \frac{D q_{\alpha}}{D t} \geq 0
$$

where $D_{i}$ is the specific dissipation, $\psi$ the specific Helmholtz free energy and $D q_{\alpha} / D t$ stands for the substantial, material time derivative of the state variables moving with the elastic reference volume. There is an emergence of localization out of the evolving dissipative structure if the absorption of power for at least one independent variable $D_{i}=0$, e.g. if we just consider the state variable temperature $T$, neglecting any other instability, and formulate a criterion for a sufficiently small time interval $\Delta t$ we find a critical temperature rise $T_{\text {crit }}$ due to shear heating minus the elastic coupling term (also known as the isentropic energy) by thermal-elastic adiabatic volume change

$$
\sigma_{i j} \dot{\varepsilon}_{i j}=\frac{1}{\Delta t}\left(\rho c_{p} \Delta T_{\text {crit }}-\alpha \Delta T_{\text {equ }} \Delta p\right)
$$

where $\alpha$ is the linear coefficient of thermal expansion, $\Delta T_{\text {equ }}$ the equilibrium temperature change of adiabatic expansion/compression and $\Delta p$ the associated pressure change. Note that $T_{\text {crit }}$ is not equivalent to $T_{c}$ defined in Eq. (1); while the former refers to the actual local temperature rise due to shear heating, giving a threshold value for the nucleation of shear bands, the latter refers to the ambient temperature, which is prone to thermal-mechanical instabilities provided that the stress is high enough. Obviously one criterion transforms into the other by acknowledging the fact that the maximum dissipation is expected for the lowest ambient temperatures that allow dislocation creep. The relation between the two temperatures will be illustrated in the numerical experiments.

The critical temperature rise for nucleation of a shear zone in the absence of thermal-elasticity (i.e. without the second term on the right hand side of Eq. (4)) is of the order of $1 \mathrm{~K}$, which is very difficult to achieve under geological conditions. In the more realistic case with thermal-elasticity, Eq. (4) shows that the contribution by the isentropic power can be significant. It effectively reduces the required temperature rise by shear heating to only a fraction of a degree, thus opening the possibility of thermal-mechanical feedback to all geological materials and not just the materials with a high activation enthalpy like olivine. All of the calculations presented here incorporate the thermal-elastic term, which thus acts as a booster to thermal-mechanical instabilities. For a comparison of calculations with and without thermal expansion refer to figure 6 in Regenauer-Lieb and Yuen (2004).

For creep processes that depend primarily on the state variable temperature the thermodynamic approach is a very natural one to pursue. It is, however, very difficult to follow the energy fluxes where the state variable pressure controls the rheology. Two approaches have been formulated so far, one based on a damage mechanics approach (Lyakhovsky, 2001) and the other based on a two-phase flow approximation (Bercovici and Ricard, 2003). It is fair to say that both approaches are still under development, in particular concerning their multi-scale behaviour and benchmarking with real rock experiments. We therefore discuss in the following a very simplified approach to couple brittle and ductile rheologies. The approach basically deals with the brittle rheology as a perfectly plastic material and thus omits any dynamics that we know is present in the brittle layer. The conclusion that we draw in terms of coupling is therefore biased by the poor knowledge of the thermodynamics of the brittle layer.

\section{Simple Unified Brittle-Ductile Model}

The purely brittle part of the lithosphere contains its weakest top layer. It is known to form highly localised modes of deformation in the form of fault/shear zones relying on the pressure dependence of the brittle yield phenomenon. The physical appearance of brittle shear zones is very similar to the ductile ones. Although there is an analogy of brittle- ( $p$-dependent) versus ductile (involving $T$-dependent) instabilities the dynamical modes of ductile instabilities are often neglected as the source for influencing and triggering the genesis of brittle earthquakes. The goal for a unified model would be to come up with a formulation for the strongest layer of the lithosphere, that allows self consistent weakening in the area where $T$-dependent and $p$-dependent processes overlap.

This strong zone is sometimes called the semi-brittle regime (Kohlstedt et al., 1995). Weakening of the semibrittle regime can occur either through thermal-mechanical instabilities (shear heating, grain size reduction, chemical reaction etc.) or through brittle dilatancy. Unfortunately, 
it is very difficult to formulate a self-consistent formulation of the fluxes of the free energies for brittle weakening. This is because it requires accurate tracking of the surface energy stored in each individual dilatant micro-crack. Hence, a common approach to dilatancy in the brittle field is the empirical Mohr-Coulomb approach introducing a quantity named the "angle of dilatancy". However, there is no unique way for turning the dilatancy angle into surface energy. Initially, we therefore do not want to mix this empirical approach with a full thermodynamic approach. Ultimately, we would want to, however, reproduce basic features of the empirical Mohr-Coulomb style approach with an approach that relies on the basic physics of tracing the energy fluxes within and outside the shear zone. For this it is necessary to first explore the system without dilatancy.

Another problematic issue of the Mohr-Coulomb approach is that it suffers from inherent mesh-dependency. This problem does not occur for fully ductile instabilities since modelling is already based on a thermodynamic (energy) concept and a physically based characteristic thermal diffusion scaling length leads to mesh independent results, if a numerical discretization criterion (based on the local Peclet number) is fullfilled (Regenauer-Lieb and Yuen, 2004). Hence, we choose to ignore in our first thermodynamic approach any of the possible brittle weakening effects. This is clearly a weakness of the method, which shall be addressed in future work. In terms of feedback this implies that we uncouple the loop, in a first order approximation, by ignoring $p$-dependent instabilities. We damp these instabilities by treating the brittle layer as a von Mises plastic body where the magnitude of the yield stress $\sigma_{y}$ grows with the pressure.

$$
\sigma_{y}=\mu p
$$

the pressure $p$ is defined by the trace of the stress tensor (positive for compression), also known as its first invariant. For simplicity the friction coefficient $\mu$ is assumed to be 1 assuming no initial cohesion. Pressure is hence used to scale the radius of the von Mises yield envelope. For the purely plastic strain rate we are thus turning the von Mises cylinder into a cone having the origin at zero. The yield envelope $\Phi$ is still, like in the Drucker-Prager solid, defined by the second invariant $J_{2}$ of the deviatoric stress tensor (primed)

$$
\Phi=\sqrt{\frac{3}{2} \sigma_{i j}^{\prime} \sigma_{i j}^{\prime}}-\mu \sigma_{y}=J_{2}-\mu p
$$

and the classical co-axial Levy-Mises flow rule is used.

$$
\dot{\varepsilon}_{i j}^{p l}=\lambda \sigma_{i j}
$$

where the superscript $p l$ refers to plastic strain-rate and $\lambda$ is a scalar multiplier with dimension $\left(\mathrm{s}^{-1} \mathrm{~Pa}^{-1}\right)$ which is zero when the stress state is below the yield stress and positive above the yield stress. In our case the material is allowed to be perfectly plastic (negligible hardening).

By selecting the von Mises plastic body for the brittle response we only allow shear cracks (Mode 2) to develop. This appears to be typical for the semi-brittle regime (Hirth and Tullis, 1994; Kohlstedt et al., 1995). We emphasize here again that we use a very crude model for the brittle layer, ignoring the possible effect of dilatant (Mode 1 crack) deformation. However, as explained above the novel thermodynamic approach and the presently available data, does not yet allow a micro-crack assessment of energy fluxes for the brittle-ductile transition (Regenauer-Lieb and Yuen, 2003). The model defined here has the advantage of a complete solution to the coupled energy equation.

In a unified model the brittle-ductile interface should hence be evolving dynamically together with the local pressure, temperature and strain-rate. Furthermore, the primary ductile creep mechanisms should not be assigned but activated by their dominance, which in turn depends on the current thermodynamic state. The classical hypo-elastic additive strain rate split is our key assumption for allowing this state dependent activation.

$$
\dot{\varepsilon}_{i j}^{\mathrm{tot}}=\dot{\varepsilon}_{i j}^{\mathrm{el}}+\dot{\varepsilon}_{i j}^{\mathrm{pl}}+\dot{\varepsilon}_{i j}^{\mathrm{cr}}
$$

The total strain rate thus results from adding elastic (including thermal-elastic), brittle (plastic) and all potential creep strain rates. In effect this is a Maxwell visco-elastic body extended to include the effect of plasticity and non-linear viscosity. In previous analyses we split the nonlinear creep processes further by adding Peierls, power law, diffusion creep strain rates (Regenauer-Lieb et al., 2001). This degree of sophistication is not warranted here since we try to formulate the simplest model possible in order to extract the basic physics of brittle-ductile coupling. This simple model produces a brittle/semi-brittle transition and a semibrittle/ductile transition without any additional assumption (Kohlstedt et al., 1995). Note that the effect of weakening through thermal-mechanical feedback is, however, a crucial ingredient for the appearance of a finite width of the semibrittle regime. We will discuss this point in further detail by comparing olivine and quartz rheology.

For defining the creep processes we assume that they comprise just the Peierls and the power law creep. We simplify further and assume that the Peierls stress only controls the onset of creep at an initial strain rate above which the power law regime is active.

$$
\dot{\varepsilon}_{\text {init }}^{\text {cr }}=\varepsilon_{o} v_{H} \exp \left(-\frac{H^{\text {Peierls }}}{R T}\right)
$$

where $R$ is the universal gas constant, $T$ the temperature and $H^{\text {Peierls }}$ is the activation enthalpy for moving a dislocation forward in the Peierls regime, $\epsilon_{0}$ is the associated strain and $v_{H}$ the frequency of a lattice vibration in a Helmholtzian framework. The value of the state dependent critical strain rate, is a fundamental material property governing the actual spatial position of the brittle/semi-brittle transition. This point has been overlooked in the previous literature. We will come back to it in the discussion. The associated initial yield stress has a first-order influence on the spatial position of the brittle/semi-brittle transition. A higher initial strain rate obviously causes higher stresses, hence a deeper brittle/semi-brittle transition. A side effect of this is that the higher energies significantly boost the potential for thermal-mechanical feedback.

For simplicity, we take conditions which do not favour the thermal-mechanical feedback mechanism by assuming, 


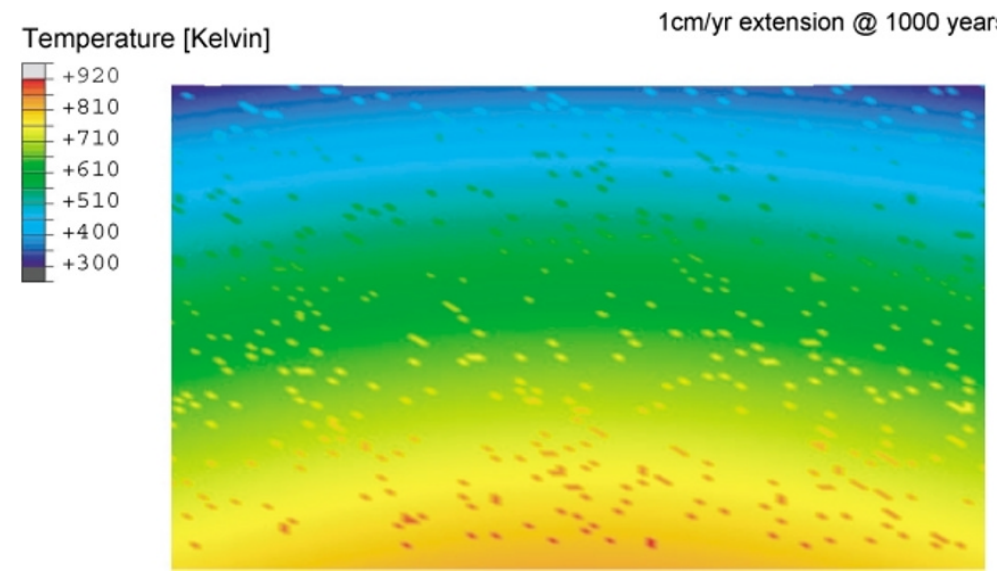

Fig. 1. Model setup of a $30 \mathrm{~km} \times 50 \mathrm{~km}$ granitic crust which has free slip at the top and bottom. The model is subject to a constant extension of $1 \mathrm{~cm} / \mathrm{yr}$. A $50 \mathrm{~km}$ long positive amplitude thermal perturbation as well as a random nodal perturbation are assumed. This starting thermal model is shown after 1000 years extension. The thermodynamic state model implies that at the same depth level there are different rheological responses owing to the local perturbations in temperature, pressure and deviatoric stress as well as a regional perturbation in temperature thus exploring the rich dynamics of possible brittle-ductile coupling.

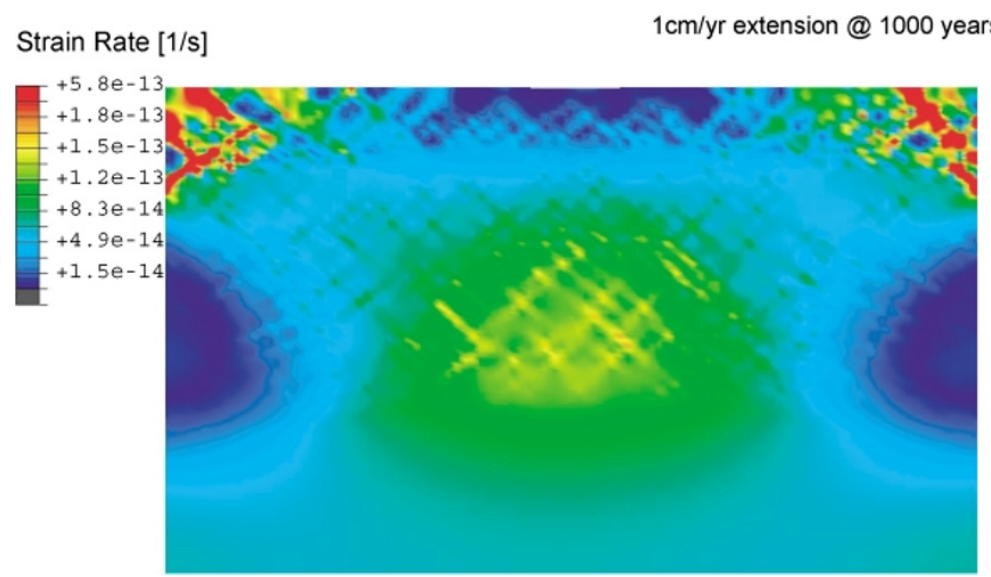

Fig. 2. Strain rate in the model (Fig. 1) after 1000 years extension. Most localization occurs in the brittle layer, close to the corner points which apparently control the model evolution. There is a clear three part subdivision into a strongly localizing top part separated by the 450-500 Kelvin isotherm from a less localizing middle part and a third smoothly deforming bottom part.

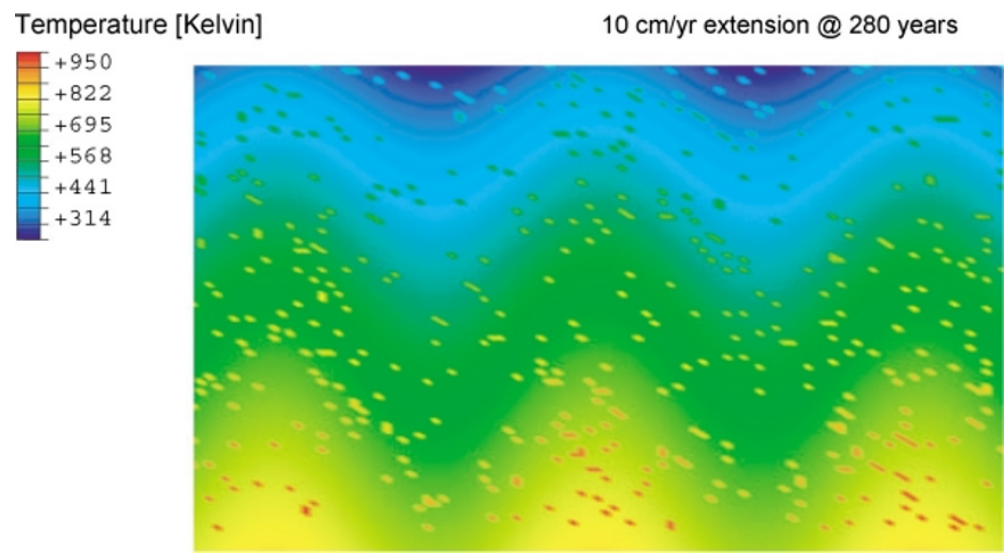

Fig. 3. The same style of model as in Fig. 1 but with a $20 \mathrm{~km}$ long wavelength thermal perturbation.

here a very low constant state independent critical initial strain rate of $10^{-16} \mathrm{~s}^{-1}$ which is not state dependent. We restrict ourselves to just using the power law creep mechanism above this strain rate.

$$
\dot{\varepsilon}_{i j}^{\mathrm{cr}}=A \sigma_{i j}^{\prime} J_{2}^{n-1} \exp \left(-\frac{H^{\text {Power }}}{R T}\right)
$$

We assume a wet crust for assessing the maximum amount of contribution by creep. We therefore use a flow law of wet quartzite where $0.4 \% \mathrm{wt}$ water has been added in a sealed capsule (Kronenberg and Tullis, 1984). The material constant $A=3.98 \times 10^{-21} \mathrm{~Pa}^{-n} \mathrm{~s}^{-1}$, the activation enthalpy is $H^{\text {Power }}=134 \mathrm{~kJ} / \mathrm{mol}$ which is in fair agreement with ex- 
Strain rate $[1 / \mathrm{s}]$

$10 \mathrm{~cm} / \mathrm{yr}$ extension @ 280 years

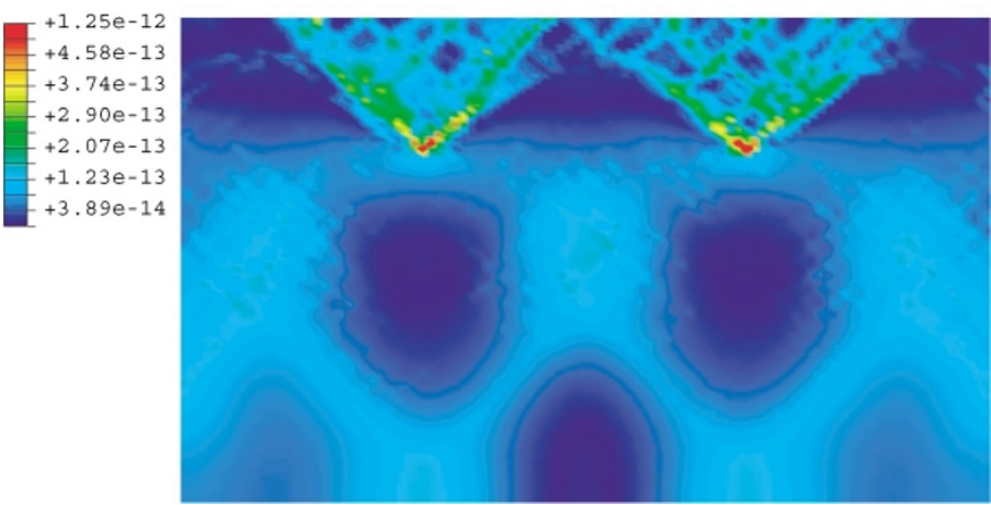

Fig. 4. The strain rate map shows a clear nucleation of brittle shear zones on the basis of the long wavelength thermal perturbations and no longer shows the affinity to nucleate on the corner points as in Fig. 2. The ten-fold increase in velocity also causes some creep localisation in the bottom layer as opposed to smooth deformation in Fig. 2. A very similar result is obtained by an increase in activation energy instead of an increase in velocity. This has been tested for dry albite rheology.

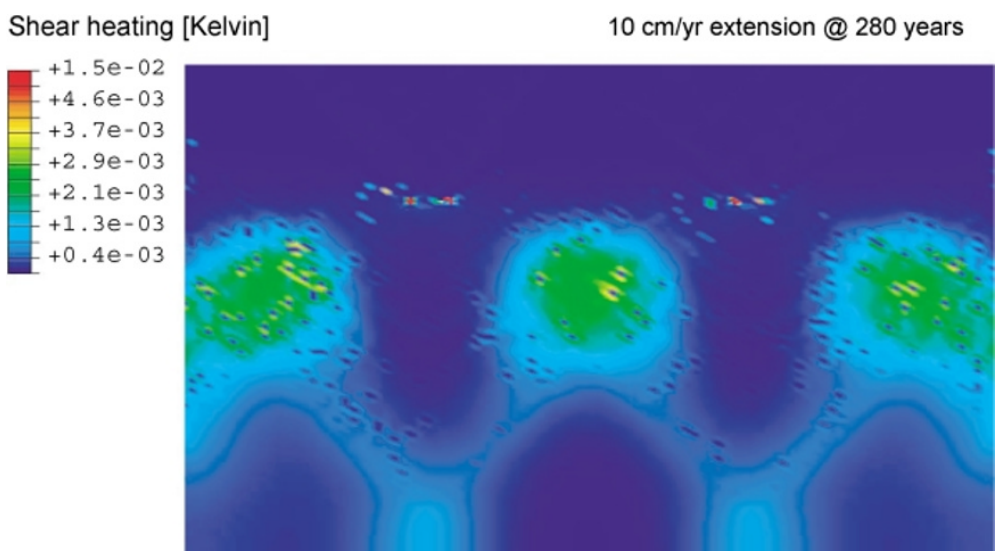

Fig. 5. Shear heating of 0.015 Kelvin (red dots) breaches the energy threshold for instability and initiate brittle shear zones shown in Fig. 4 . The red dots themselves only show the dissipated work due to thermal feedback. Feedback is clearly nucleating on the random perturbations in the middle layer of the model. This calculation shows the enormous impact of the thermal-elastic feedback term in Eq. (4). Without this term the model has to be stretched much longer before shear zones can nucleate but the phenomenon is the same.

Strain Rate [1/s] @ 1 Myr
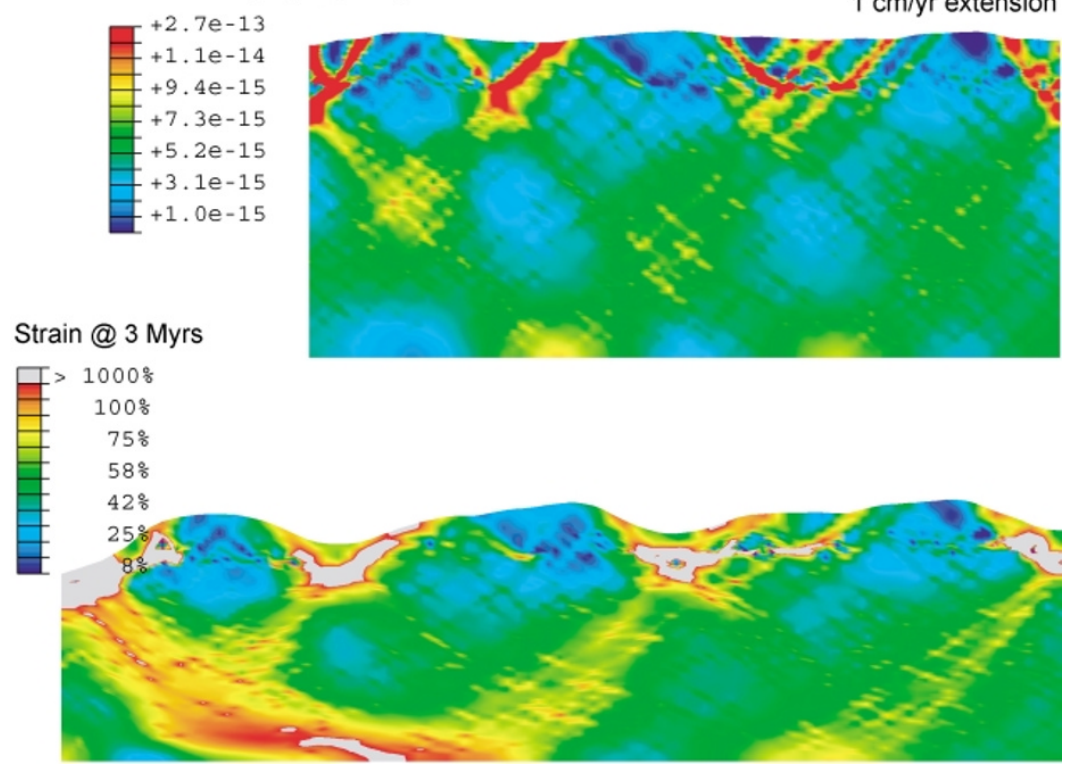

Fig. 6. Top: strain rate of wet quartzite crust after 1 Ma extension. Bottom: strain of the same crust after 3 Ma extension. The brittle/semi brittle interface reaches a maximum detachment strain of $2900 \%$ after $3 \mathrm{Ma}$ (shown in grey). However, for quartz rheology we observe significant fault splays into the semi-brittle layer below the detachment fault. The rheological layering of a brittle, brittle/ductile and purely ductile layer is maintained during extension and effectively leads to a complete necking of the brittle layer during extension. 
trapolations from naturally deformed samples (Hirth et al., 2001) while the power law exponent of $n=2.6$ is probably reflecting a strong contribution of dislocation glide which is important at low temperatures in the semi-brittle transition area where brittle and ductile deformation overlap (Hirth et al., 2001; Hirth and Tullis, 1994). The particular flow law chosen should thus provide a good compromise for investigating brittle-ductile coupling.

\section{Model Formulation and Sensitivity Analysis}

The model is formulated for a generic a $50 \mathrm{~km}$ long, 30 $\mathrm{km}$ deep crustal section of the above defined wet quartzite. This model has been subject to pure shear extension boundary conditions $(1 \mathrm{~cm} / \mathrm{yr})$ at the right boundary while the left boundary has been held fixed. An initial continental geotherm been assumed equivalent to $70 \mathrm{mWm}^{-2}$ surface heat flow with a $10 \mathrm{~km}$ thick exponentiall decaying radiogenic layer as well as a mantle contribution of $30 \mathrm{mWm}^{-2}$ flowing in from the bottom. It can be shown that without the presence of feedback processes this model configuration should provide homogenous pure shear extension (Shawki, 1994). We perturb this model by two different thermal perturbations. One is a random thermal perturbation on the nodes. This induces local thermal strain due to thermal expansion and weakens the rheology locally, thus mimicking heterogeneity in a real rock. The implication for symmetry breaking as well as a more detailed description of the motivation for this model setup is described elsewhere (Regenauer-Lieb and Yuen, 2004). The second perturbation is a sinusoidal perturbation which we use in the first sensitivity analysis to test the effect of long wavelength lateral temperature contrasts.

The amplitude of the thermal perturbation in Fig. 1 is greatly exaggerated compared to a real rock for the purpose of a sensitivity analysis. We want to test which state variable controls the nucleation, spacing and width of brittle and ductile faults. We show in Fig. 2 the strain rate map of this calculation and clearly see a three part horizontal stratification following the long wavelength perturbation. The top domain is the brittle layer where only p-dependent localization occurs. The middle layer shows an area where chiefly T-dependent localization occurs and the bottom part is the area where smooth ductile creep occurs without localization phenomenon. We can also clearly see that the brittle localization is very much controlled by the box boundaries, which translates down to the ductile layer. This is not an ideal case for further investigation but is an illustration how the geometrical singularities at surface (the corner points) are communicated downwards into the ductile crust. This model clearly shows the potential control of the geometry of the surface on faulting in the ductile crust. We proceed in a model that has a shorter wavelength perturbation and also test the influence of a very high extension velocity of $10 \mathrm{~cm} /$ year. The thermal model is shown in Fig. 3 and its associated strain rate map is shown in Fig. 4.

In Fig. 4 it is clear that the brittle faults are no longer nucleating on geometrical singularities but on thermal perturbations. We will now investigate the reason for the nucleation of shear zones in more details. For this we use the thermodynamic criterion and look at the shear heating occurring in the model. The total amount of mechanical energy dissipated in the first 280 years of extension is shown in Fig. 5.

This shows that in the absence of geometric imperfections there is a clear thermal control on nucleation of brittle shear zones. Having discussed the fundamental "ductile" control for the nucleation of brittle shear zones, which is clearly evident in the experiment shown in Figs. 3, 4 and 5, we now discuss the spacing of the shear zones. We emphasize as a caveat that we do not intend to give a full discussion of the spacing of the folding/necking problem, which clearly involves two fundamental scales a bifurcation analysis. One scale is dictated by the shear bands (material scale) and the other scale by the rate of growth of geometric imperfections (classical folding/necking analysis dealing with the fastest growing geometric imperfection). In geology we observe both phenomena and a thorough analysis of the faulting/folding problem is clearly still lacking (Steif, 1987). In our case the necking phenomenon is controlled by the nucleation of shear zones.

For a faulting control the thermodynamic approach also gives a very simple answer. We have observed in previous work (Regenauer-Lieb and Yuen, 2004) that the system of multiple interacting ductile faults is attracted to the lowest frequency Eigenmode of the system. In analogy to the Euler buckling problem the lowest frequency Eigenmode is the lowest possible energetic mode. This implies that the system converges after sufficient deformation into the largest stable fault system that can be achieved. This fault system is controlled by the thickness of the brittle layer, which necks, as we will show later, on a subhorizontal detachment as indeed postulated by Ord and Hobbs (1989). This thermalmechanical weakening has the effect that we are stretching the brittle layer like an isolated layer with a free top and bottom surface. For our rheology this resolves in a reflection of faults at roughly $45^{\circ}$ angle thus giving a characteristic spacing of twice the layer thickness as the starting conditions. Although we are moving during continuing deformation into more geometric complexity the weakening on the initial detachment still governs the final spacing. For a more realistic brittle rheology with dilatant material behaviour the spacing is expected to be somewhat shorter but the basic conclusion is still valid if the nucleation is controlled by thermal-mechanical feedback.

\section{Width of the (Semi-Brittle) Detachment Layer}

The final discussion aims at giving a simple description of the width of the detachment layer for listric faulting and the tendency of the material to develop a subhorizontal detachment layer. For this we compare extreme end member rheologies, i.e. listric faulting for wet olivine and wet quartzite. The model for wet quartzite is a more geologically relevant version of the extremely perturbed model shown in Figs. 4 and 5. We reduce the sinusoidal temperature perturbation to 5 degrees amplitude and lower the velocity boundary condition back to an extension velocity of $1 \mathrm{~cm} / \mathrm{yr}$ (Fig. 6). The extreme thermal sensitivity shown in Fig. 6 for the nucleation of brittle faulsts has the effect that the small temperature perturbation on the lowest frequency Eigenmode still controls faulting of the brittle layer. 


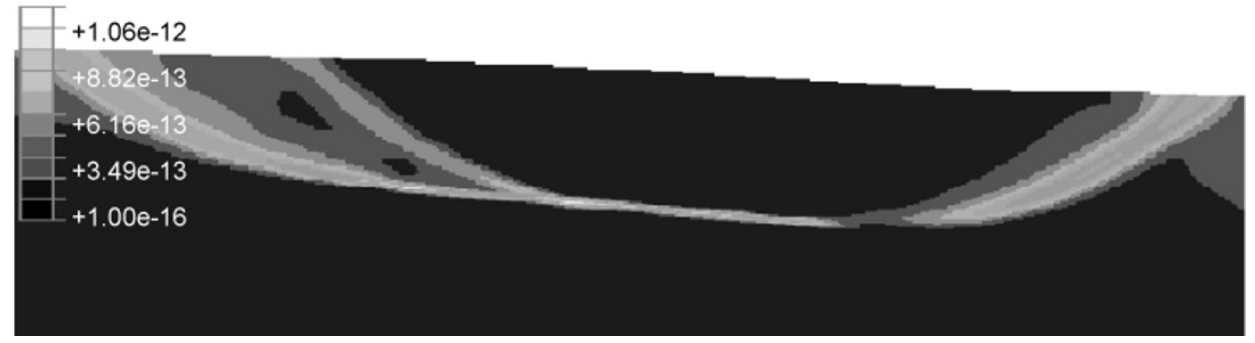

Fig. 7. Extension of a $70 \mathrm{~km}$ thick oceanic slab with free slip at top and bottom, a small amount of bending deformation (tilt) is superposed. A listric fault system develops with a decoupling horizon at around $15 \mathrm{~km}$ depth for olivine rheology. The maximum in plane principal strain rate ( $\mathrm{s}^{-1}$ ) is shown contoured. Note the high degree of localization compared to quartz and magnitude of strain-rate on the decoupling horizon.

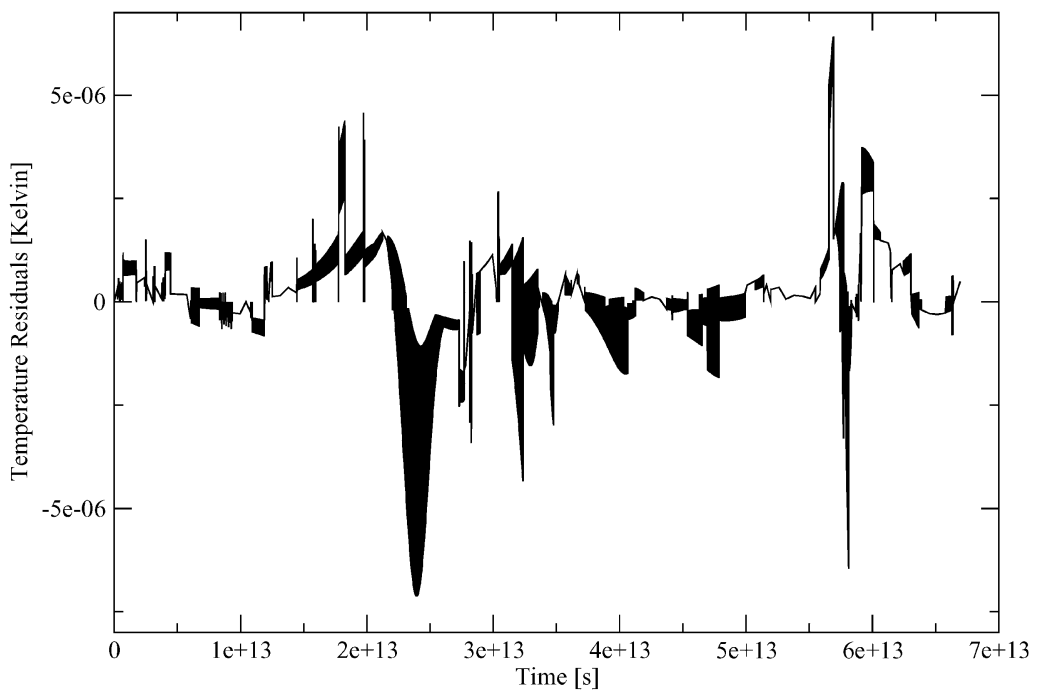

Fig. 8. Convergence characteristics of the quartz rheology calculation traced over a 2 Ma time frame. The residuals of the temperature show that we clearly have enough resolution for detecting a fraction of a degree shear heating. The small residuals have been achieved by dynamically reducing the time step down to a magnitude of less than a year $\left(10^{7} \mathrm{~s}\right.$ oscillations result in a filled black curve $)$ and damping the solution by the restriction that no velocity larger than the far field boundary condition is allowed.

Although brittle faults turn listric onto a subhorizontal detachment layer there is still a lot of complexity below the detachment. However, we do not yet observe much of a symmetry breaking which is characteristic for ductile thermal-mechanical instabilities (Regenauer-Lieb and Yuen, 2004). The brittle crust, although in our modelling formulation entirely passive (no weakening), acts like a damping regulator to thermal-mechanical instabilities below. Nonetheless, we clearly see the beginning of asymmetric deformation below the brittle crust in oblique branches propagating into the lower crust outside the detachment layer down to about $20 \mathrm{~km}$ depth. This tendency for asymmetry has fully propagated upwards through the detachment layer after $3 \mathrm{Ma}$ of deformation at which point the detachment has experienced $>1000 \%$ strain (Fig. 6) and the ductile crust is almost exposed to the surface on the sites of necking instabilities.

The semi-brittle feedback layer where discrete ductile faulting occurs below the detachment has a width of about $10 \mathrm{~km}$ from $(450-650 \mathrm{~K})$, which is remarkably thicker than the width of the feedback layer for olivine (500 m width at the $875 \mathrm{~K}$ isotherm for $498 \mathrm{~kJ} / \mathrm{mol}$ activation energy). It should be emphasized that the pure shear boundary conditions that trigger listric faults in quartz and feldspar rheology are not sufficient for the case of olivine. In pure shear the crust either keeps a stiff elastic core for dry conditions or ruptures entirely (Regenauer-Lieb et al., 2004). However, when performing the same extension experiment with a $70 \mathrm{~km}$ thick olivine lithosphere and superposing a small component of bending the wet olivine model can go into listric faulting with a distinct narrow detachment faults as shown in Fig. 7.

The rich dynamics of quartz as opposed to olivine is clearly visible in the energy residuals that trace the convergence behaviour of the energy equation. These residuals are shown in Fig. 8.

The same model boundary conditions applied to olivine rheology (compare figure 12 of Regenauer-Lieb and Yuen, 2004) gives larger magnitude feedback owing to the activation energy term (Eqs. (1) and (2)) but a much more steady long term dynamics owing to the rapid convergence of olivine towards few ductile faults. In quartz multiple interacting ductile faults are maintained on the other hand until the formation of a metamorphic core complex (high 


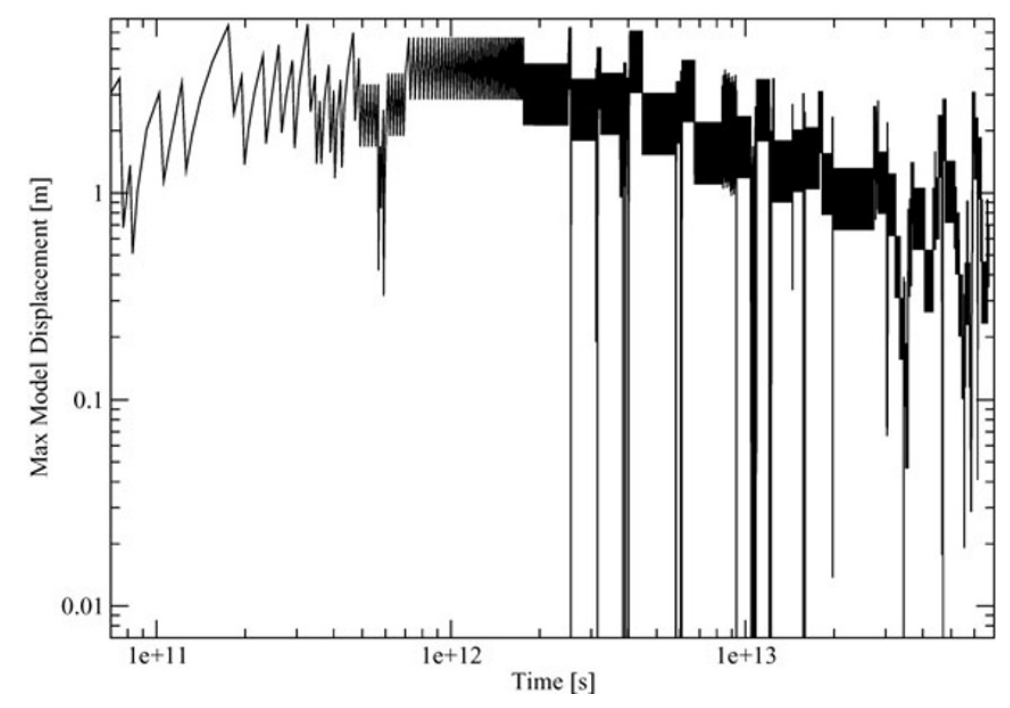

Fig. 9. Maximum model displacements. The oscillations are damped by the kinematic restriction of the far field boundary. Quartz rheology has a very rich dynamics compared to olivine rheology.

strain grey area in Fig. 6) has been completed. The log-log plot in Fig. 9 shows some more details of the oscillations in terms of the maximum displacements during an individual timestep.

\section{Summary and Discussion}

We have discussed a first simple thermodynamic model of the brittle/semi-brittle and semi-brittle/ductile transition. The approach is simplified because it only contains thermalmechanical feedback by shear heating and thermal elasticity. It ignores the stored energy in microcracks. The approach is also simplified because it parameterizes the effect of low temperature plasticity into a very low critical strain rate for initial creep. Within this simple mode we have shown that a homogeneous granitic crust in tension self-consistently develops instabilities on a subhorizontal layer within the crust which upon further straining ultimately leads to uplift and exposure of the ductile lower crust to the surface (building of a core complex). Within our model multiple interacting "ductile" ( $T$-dependent) instabilities trigger faulting in the "brittle" ( $p$-dependent) crust above the thermal-mechanically weakened sublayer. Our thermodynamic framework shows the tectonic control of the detachment zone below the brittle-ductile transition on the overall crustal evolution. This detachment always develops for quartz rheology, even for pure shear boundary conditions, into listric faults cutting the top brittle crust. For olivine rheology the tendency for building a detachment is somewhat lower but the instability is much more vigorous in amplitude. For olivine the nucleation of brittle faults also relies on ductile instabilities, however olivine appears to develop a much narrower detachment zone and lacks the semi-brittle interaction zone.

We attribute this lack in dynamic behaviour to the higher activation energy of olivine in both the power-and the Peierls low temperature plastic creep laws. The low temperature plastic yield phenomenon (embodied here in an initial strain rate for creep) implies a much higher ratio of low temperature yield stress over the Youngs modulus for olivine than for quartz. This property is of fundamental importance for the thermodynamics of the system. If the ratio of elasticity over yield stress is low (quartz) a semibrittle layer develops where there are significant oscillations between elastic stored energy and release of the energy in creep bursts (as shown in Figs. 8 and 9). If the ration is high the semi-brittle feedback domain almost vanishes and the flutter in stored elastic energy and creep burst reduces significantly. This important finding will be discussed in further details in a separate publication. For the present discussion we would like to point out that the initial strain-rate for creep, which is controlled by the low temperature (Peierls) yield stress, has a first order influence on the depth of the brittle/semi-brittle transition. For near adiabatic processes (negligible thermal diffusion), a constant initial strain rate for creep implies for instance that under a faster loading rate the critical energy for feedback processes is reached earlier than by a slow loading rate. However, the formation of the brittle/semi-brittle transition still roughly lies in the same spatial position because it is the critical dissipation that governs the onset of feedback.

Our calculations have been carried out to substantial strain on the detachment horizon (up to 2900\%) and full severance of the brittle crust. We would like to caution that the restrictive far field boundary conditions and the low Peierls stress, chosen for our calculations have been motivated by giving a lower bound estimate for the role of thermal-mechanical instabilities in the crust, which are likely to be more vigorous in a real crust. However, even in this restrictive environment we observe a strong tendency to unstable slip (Figs. 8 and 9) with typical periods ranging between 2 and 5 years $\left(7 \times 10^{8} \mathrm{~s}\right.$ and $\left.1.5 \times 10^{9} \mathrm{~s}\right)$. Note that higher characteristic frequencies are damped by the constant velocity boundary conditions. The lower frequency creep bursts should be ideal for monitoring preseismic build up by geodetic measurements. Future high resolution numerical models driven by more realistic, dynamic large scale geodynamic simulations of subduction (Funiciello et al., 2003; Morra et al., 2004) are clearly indi- 
cated.

Another necessary point that needs to be added for more realism is a brittle crust with more dynamics. We have pointed out that the previously suggested thermodynamic framework for the brittle crust cannot yet be extended into the scale of earthquakes. A chief problem with these approaches is that most experiments do not extrapolate to the scale of the rupture of earthquakes. The rate and state variable friction theory (Dieterich, 1979) may be an exception, but this approach is dealing with an entirely empirical description of thermodynamics of faulting. As an intermediate future step, before the ability of deriving a robust theory of friction, we suggest to study the earthquake problem by coupling a rate and state variable friction model in a continuum (Ord et al., 2004) and a fluid flow model (Hobbs et al., 2004 ) in order to zoom into the dynamics of the brittle crust. We have shown here, that for our simplified setup the brittle seismogenic layer is the slave of the ductile layer. While this result clearly reflects our simplified choice of the brittle rheology, we have nevertheless shown that in a fully coupled thermal-mechanical granitic crust ductile creep bursts, which can reach pulses of a single day, might have a significant impact on the dynamics of the brittle regime.

Acknowledgments. We would like to acknowledge first of all the Japanese initiative of Slip and Flow processes below the seismogenic region for instigating this work and inviting a crossdisciplinary group of researchers to discuss the earthquake problem on site in Japan. We were very impressed by the field exposures and the presentation, venues like these will bring the science of earthquakes forward in an unprecedented manner. Next we would like to acknowledge helpful comments by Prof. Hirahara and Dr. Susan Ellis who made sure that the manuscript is complete and also helped to put it into its right place. Amongst the many cultural experiences we owe to all our hosts we enjoyed, as an aside, the spiritual introduction into several chapters of enlightenment in the Azi Kou Bou Dining Bar.

\section{References}

Bercovici, D. and Y. Ricard, Energetics of a two-phase model of lithospheric damage, shear localization and plate-boundary formation, Geophysical Journal International, 152, 581-596, 2003.

Cherukuri, H. P. and T. G. Shawki, An energy-based localization theory: I. Basic Framework, International Journal of Plasticity, 11, 15-40, 1995a.

Cherukuri, H. P. and T. G. Shawki, An energy-based localization theory: II. Effects of the diffusion, inertia, and dissipation numbers, International Journal of Plasticity, 11, 41-64, 1995b.

Dieterich, J. H., Modeling of Rock Friction. 1. Experimental Results and Constitutive Equations, Journal of Geophysical Research, 84(NB5), 2161-2168, 1979

Fleitout, L. and C. Froidevaux, Thermal and Mechanical Evolution of Shear Zones, Journal of Structural Geology, 2(1-2), 159-164, 1980.

Funiciello, F., G. Morra, K. Regenauer-Lieb, and D. Giardini, Dynamics of retreating slabs: 1 . Insights from two-dimensional numerical experiments, Journal of Geophysical Research-Solid Earth, 108(B4), art. no.2206, 2003.

Hirth, G. and J. Tullis, The brittle-plastic transition in experimentally deformed quartz aggregates, Journal of Geophysical Research, 99(B6), 11731-11747, 1994.

Hirth, G., C. Teyssier, and W. J. Dunlap, An evaluation of quartzite flow laws based on comparisons between experimentally and naturally de- formed rocks, International Journal of Earth Sciences, 90(1), 77-87, 2001.

Hobbs, B. E. and A. Ord, Plastic instabilities: Implications for the origin of intermediate and deep focus earthquakes, Journal of Geophysical Research, 93(B9), 10521-10540, 1988.

Hobbs, B. E., A. Ord, and C. Teyssier, Earthquakes in the ductile regime, Pure and Applied Geophysics, 124(1/2), 310-336, 1986.

Hobbs, B. E., A. Ord, and K. Regenauer-Lieb, Fluid reservoirs in the crust and mechanical coupling between the upper and lower crust, Earth Planets Space, 56, this issue, 1151-1161, 2004.

Kameyama, M., D. A. Yuen, and H. Fujimoto, The interaction of viscous heating with grain-size dependent rheology in the formation of localized slip zones, Geophysical Research Letters, 24(20), 2523-2526, 1997.

Kameyama, M., D. A. Yuen, and S. Karato, Thermal-mechanical effects of low temperature plasticity (the Peierls mechanism) on the deformation of a viscoelastic shear zone, Earth and Planetary Science Letters, $\mathbf{1 6 8}$, 159-162, 1999.

Kohlstedt, D. L., B. Evans, and S. J. Mackwell, Strength of the lithosphere: Constraints imposed by laboratory measurements, Journal of Geophysical Research, 100(B9), 17587-17602, 1995.

Kronenberg, A. K. and J. Tullis, Flow strength of quartz aggregates: Grain size and pressure effects due to hydrolytic weakening, Journal of Geophysical Research, 89, 42981-42997, 1984.

Lyakhovsky, V., Scaling of fracture length and distributed damage, Geophysical Journal International, 144(1), 114-122, 2001.

Lyakhovsky, V., Y. Ben-Zion, and A. Agnon, Earthquake cycle, fault zones, and seismicity patterns in a rheologically layered lithosphere, Journal of Geophysical Research-Solid Earth, 106(B3), 4103-4120, 2001.

Meissner, R. and L. Brown, Seismic reflections from the Earths crustcomparative-studies of tectonic patterns, Geophysical Journal International, 105(1), 1-2, 1991.

Morra, G., K. Regenauer-Lieb, and D. Giardini, On the curvature of oceanic arcs, Nature, 2004 (submitted).

Ogawa, M., Shear instability in a viscoelastic material as the cause of deep focus earthquakes, Journal of Geophysical Research, 92(B1), 1380113810, 1987.

Ord, A. and B. E. Hobbs, The strength of the continental crust, detatchment zones and the development of plastic instabilities, Tectonophysics, $\mathbf{1 5 8}$, 269-289, 1989.

Ord, A., B. E. Hobbs, and K. Regenauer-Lieb, A smeared seismicity constitutive model, Earth Planets Space, 56, this issue, 1121-1133, 2004.

Regenauer-Lieb, K. and D. Yuen, Rapid conversion of elastic energy into shear heating during incipient necking of the lithosphere, Geophysical Research Letters, 25(14), 2737-2740, 1998.

Regenauer-Lieb, K. and D. A. Yuen, Modeling shear zones in geological and planetary sciences: Solid- and fluid-thermal-mechanical approaches, Earth Science Reviews, 63, 295-349, 2003.

Regenauer-Lieb, K. and D. A. Yuen, Positive feedback of interacting ductile faults from coupling of equation of state, rheology and thermalmechanics, Physics of Earth and Planetary Interiors, 142(1-2), 113$135,2004$.

Regenauer-Lieb, K., D. Yuen, and J. Branlund, The initation of subduction Criticality by addition of water?, Science, 294, 578-580, 2001.

Regenauer-Lieb, K., B. Hobbs, H. Mulhaus, A. Ord, D. A. Yuen, and S. van der Lee, Lithosphere fault zones rejuvenated, Tectonophysics, 2004 (submitted).

Shawki, T. G., An energy criterion for the onset of shear localization in thermal viscoplastic material, Part I: Necessary and sufficient initiation conditions, Journal of Applied Mechanics, 61, 530-537, 1994.

Steif, P. S., On the initiation of necking modes in layered plastic solids, Journal of Applied Mechanics, 54, 141-146, 1987.

Yuen, D. A., L. Fleitout, G. Schubert, and C. Froidevaux, Shear deformation zones along major transform faults and subducting slabs, Geophysical Journal of the Royal Astronomical Society, 54(1), 93-119, 1978.

K. Regenauer-Lieb (e-mail: regenaue@uni-mainz.de), B. Hobbs, and A. Ord 